\title{
A Proposed Method to Quantify the Effect of Head and Shoulder Position on Kyphotic Deforming Forces before and after Spine Surgery
}

\author{
Daniel Bonthius ${ }^{1 *}$, Richard Gross ${ }^{1}$, Yongren Wu ${ }^{1,2}$ and Hai Yao ${ }^{1,2}$ \\ ${ }^{1}$ College of Medicine, Medical University of South Carolina, Charleston SC, USA \\ ${ }^{2}$ Department of Bioengineering, Clemson University, Clemson SC, USA
}

\begin{abstract}
Structured Abstract
Objective: To describe a method for quantifying kyphotic deforming forces secondary to head and shoulder position, usng three illustrative cases.

Summary of background data: While sagittal balance of the spine is examined regularly before and after surgery for kyphotic spinal deformity, current radiographic measurement techniques limit assessment of sagittal balance to the spine and pelvis (or femoral heads) only and ignore the head and shoulder position. Patients with kyphotic deformity often have anterior positioning of the head, along with protracted shoulders with limited shoulder motion and weak back muscles. Anterior positioning of the head and shoulders, in relation to the upper instrumented vertebrae (UIV), produces a kyphotic deforming force that is not appreciated with current methods for calculating sagittal balance. In some patients with instrumented spines, this kyphotic force may contribute to the development of proximal junctional failure (PJF) or loss of fixation. This paper utilizes three case studies to describe a method for quantifying the kyphotic deforming force of the head and shoulders on the spine.
\end{abstract}

Materials and methods: Simple force vectors derived from anatomical landmarks on the head and shoulders are used to calculate kyphotic deforming moments on the spine. In general, the calculation for a moment $(\mathrm{M})$ around a point involves multiplying a force (F) by the distance (d) of the applied force from the point of interest $(M=F \cdot d)$. Three patients with kyphosis underwent instrumentation for their deformities. Lateral radiographs and imaging software were used to determine the distance (in $\mathrm{cm}$ ) of the head (external acoustic meatus) and shoulders (center of the humeral head) from the center of the vertebral body at the apex of the curve pre-operatively, and the superior instrumentation at the upper instrumented vertebrae post-operatively. This distance was multiplied by the weights of the head and shoulders (estimated as percentages of body weight) to calculate the kyphotic bending moments $\left(M_{\text {total }}\right)$, expressed as $N \cdot c m$.

Results: The average pre-operative kyphotic deforming force on the apex due to head and shoulder position was 1114.0 $\pm 386.0 \mathrm{~N} \cdot \mathrm{cm}$. Surgical correction of the spinal deformity decreased this force, as the post-operative deforming force on the upper instrumented vertebrae was $720.2 \pm 321.4 \mathrm{~N} \cdot \mathrm{cm}$ immediately post-operatively and further decreased to $597.6 \pm 223.6 \mathrm{~N} \cdot \mathrm{cm}$ at final follow-up. Only one of the three patients experienced proximal junctional failure and loss of fixation. Unlike the other two cases, this patient had a persistently high kyphotic bending moment $\left(\mathrm{M}_{\text {total }}\right)$ and anterior positioning of his head and shoulders that did not improve with surgery. This persistent kyphotic force likely contributed to his complications. Calculation of pre-operative and post-operative kyphotic deforming forces provided the surgeon with a quantitative assessment of forces acting on the spine and instrumentation.

Conclusions: Quantifying the kyphotic deforming forces produced by the head and shoulder position is simple and informative. Calculation of these forces may be useful to surgeons pre-operatively and post-operatively. This method may be used, in part, to predict the likelihood of post-operative complications, as the persistence of high kyphotic deforming forces suggest a higher risk for proximal junctional failure and loss of fixation.

Level of Evidence: IV

Keywords

Kyphosis, Shoulder; Head, Scapular protraction, Proximal junctional failure

Copyright: (C) 2021 Bonthius D, et al. This is an open-access article distributed under the terms of the Creative Commons Attribution License, which permits unrestricted use, distribution, and reproduction in any medium, provided the original author and source are credited. 
Citation: Bonthius D, Gross R, Wu Y, et al. (2021) A Proposed Method to Quantify the Effect of Head and Shoulder Position on Kyphotic Deforming Forces before and after Spine Surgery. J Orthop Surg Tech 4(1):324-330

\section{Introduction}

Posterior spinal instrumentation and fusion is a common procedure for correction of severe spinal deformities. This procedure involves the placement of metal rods anchored to the spine proximally and distally with pedicle screws or hooks, to align the spine and correct the deformity. Excellent results have been achieved for scoliotic coronal plane deformities. However, for kyphotic sagittal plane deformities, the results are often compromised by high complication rates [1-4]. Two common complications are proximal junctional failure (PJF) and loosening or loss of fixation, especially at the proximal fixation site $[5,6]$. Proximal junctional failure is a progressive localized kyphosis that develops just superior to the proximal fixation site. It has a reported incidence ranging from $5.8 \%$ to $59 \%$, depending on the patient population, with higher rates observed in elderly patients [7]. Loss of proximal fixation includes loosening or pullout of pedicle screws or hooks at the proximal fixation site and is especially common in patients with kyphosis and osteoporosis [8].

Several factors that may cause or contribute to these complications have been reported [5-7]. However, one factor that has not yet been examined is the role of head and shoulder position in sagittal balance and the role that this may play in proximal junctional failure and loss of fixation. The currently employed technique for measuring global sagittal balance involves drawing a vertical plumb line from the $\mathrm{C} 7$ vertebral bodyon lateral radiographs and measuring sagittal offset from either the sacral plate or the femoral head [8]. While global sagittal balance of the spine is examined regularly after surgery, this technique limits assessment of sagittal balance to the spine and pelvis only, and ignores the head and shoulder position. The T1-pelvic angle is an alternative measurement of sagittal balance that does incorporate increased flexion of the upper thoracic spine, but likewise does not incorporate forces on the spine secondary to head and shoulder position [9].

Patients with kyphotic deformity often have anterior positioning of the head, along with protracted shoulders with limited shoulder motion and weak back muscles [10-12]. Anterior positioning of the head and shoulders, relative to the upper instrumented vertebrae (UIV), produces a kyphotic deforming force that is not appreciated with current methods for calculating sagittal balance. In patients with excessive anterior positioning of the head and protracted shoulders, this kyphotic deforming force may contribute to the development of proximal junctional failure or loss of fixation. Clinically, these complications are observed at high rates in patients that fit this phenotype. Thus, it is critical to develop a radiographic measurement technique to assess sagittal balance of the head and shoulder position, relative to the spine.

This paper utilizes three case studies to describe a method for quantifying the kyphotic deforming force of the head and shoulders on the spine. Measurements are based on pre-operative and post-operative position of the head and shoulders on lateral radiographs, and the force is expressed as a bending moment at a point on the spine. Pre-operatively, this information can be helpful in planning surgical strategy to minimize post-surgical complications. Post-operatively, the method can be used to supplement previous sagittal balance calculations by providing a measurement that includes the contribution of head and shoulder position and weight to kyphotic deforming forces.

\section{Materials and Methods}

Three pediatric patients who underwent surgery for kyphosis are described. Kyphotic deforming forces resulting from head and shoulder position, relative to the spine, were calculated as bending moments pre-operatively and post-operatively for each patient. Kyphotic bending moments were calculated at the apex of the deformity pre-operatively and at the UIV (upper instrumented vertebrae) post-operatively, as the deforming forces are concentrated at this site.

The calculation for a moment $(\mathrm{M})$ around a point involves multiplying a force (F) by the distance (d) of the applied force from the point of interest $(M=F \cdot d)$. In this study, force is the force of gravity (weight) of the head and shoulders (and arms). The line of action of this force is downward and perpendicular to the plane of the floor. The weights of these body segments (in kg) were calculated based on their estimated percentage of total body weight. Previous studies have documented that the weight of the head is approximately $7.1 \%$ of the total body weight, while the weight of both shoulders and arms is approximately $10.4 \%$ of total body weight [13]. Lateral radiographs and Impax $6.5^{\mathrm{TM}}$ imaging software were used to determine the distance (in $\mathrm{cm}$ ) of the head (external acoustic meatus) and shoulders (center of the humeral head) from the center of the vertebral body at the apex of the curve pre-operatively and the superior instrumentation at the upper instrumented vertebrae post-operatively. This distance was multiplied by the weights of the head and shoulders (as percentage of body weight) to calculate the kyphotic bending moments, expressed as $\mathrm{N} \cdot \mathrm{cm}$ (Figure 1). The moment due to the weight and position of the head is expressed by the equation $M_{h}=W_{h} \cdot L_{h} \cdot 9.8$ (Equation 1), where $W_{h}$ is the weight of the head (in $\mathrm{kg}$ ) as a percentage (7.1\%) of total body weight, and $L_{h}$ is the distance (in $\mathrm{cm}$ ) from the external acoustic meatus to the apex of the deformity pre-operatively and upper instrumented vertebrae post-operatively. The moment due to the weight and position of the shoulders is expressed by the equation $\mathrm{M}_{\mathrm{s}}=\mathrm{W}_{\mathrm{s}} \cdot \mathrm{L}_{\mathrm{s}} \cdot 9.8$ (Equation 2), where $\mathrm{W}_{\mathrm{s}}$ is the weight of the shoulders and arms (in $\mathrm{kg}$ ) as a percentage (10.4\%) of total body weight, and $L_{h}$ is the distance (in $\mathrm{cm}$ ) from the center of the humeral head to the apex of the deformity pre-operatively and upper instrumented vertebrae post-operatively.

*Corresponding author: Daniel Bonthius, College of Medicine, Medical University of South Carolina, 441 Meeting St. Apt 415, Charleston SC, 29403, USA, Tel: 319-325-9805

Accepted: June 08, 2021

Published online: June 10, 2021

Citation: Bonthius D, Gross R, Wu Y, et al. (2021) A Proposed Method to Quantify the Effect of Head and Shoulder Position on Kyphotic Deforming Forces before and after Spine Surgery. J Orthop Surg Tech 4(1):324-330 


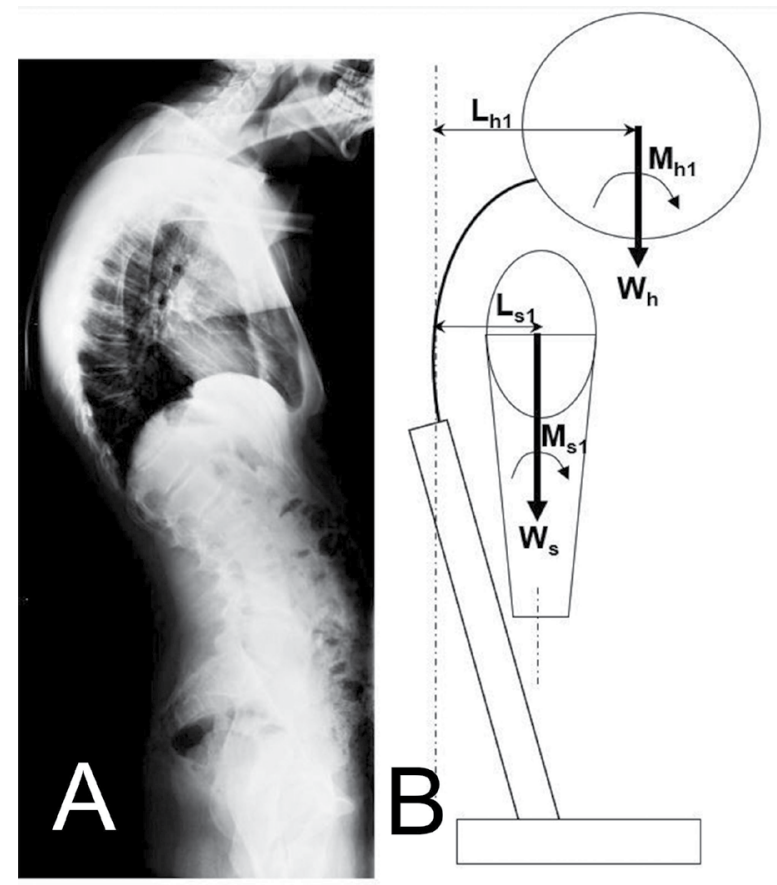

$$
M_{\text {total }}=M_{h}+M_{s}=\left(W_{h} \cdot L_{h} \cdot 9.8\right)+\left(W_{s} \cdot L_{s} \cdot 9.8\right)
$$
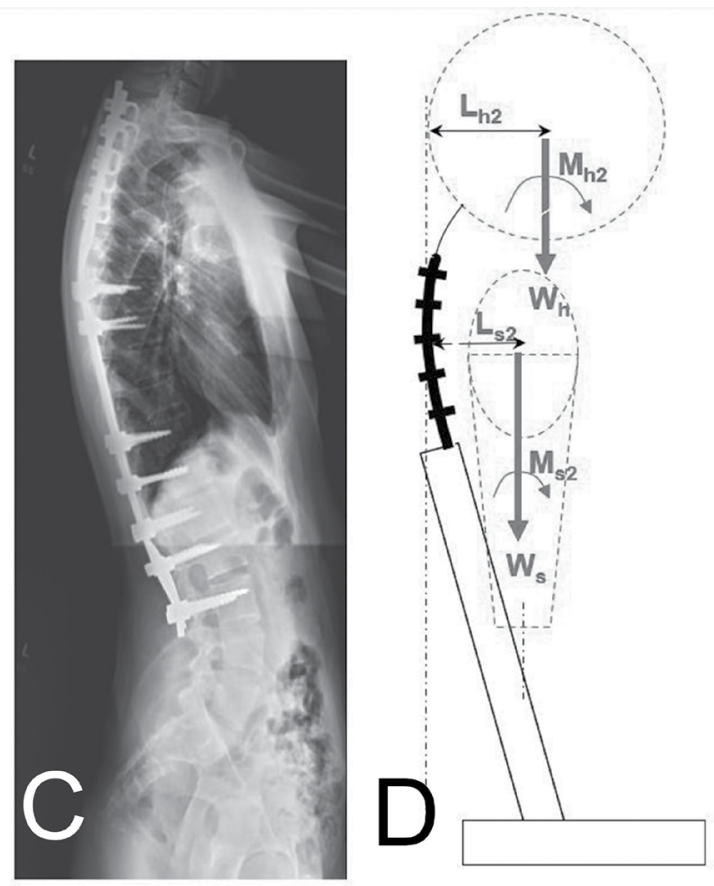

$M_{\text {total }}=M_{h}+M_{s}=\left(W_{h} \cdot L_{h} \cdot 9.8\right)+\left(W_{s} \cdot L_{s} \cdot 9.8\right)$

Figure 1: Schematic view of a biomechanical analysis on (A and B) a patient with kyphosis prior to surgery, and (C and D) fusion with posterior instrumentation and pedicle screw fixation. $L_{h}$ and $L_{s}$ : Distance from head (external acoustic meatus) and shoulder (center of humeral head) to the center of the vertebral body at the apex of the deformity pre-operatively and the upper instrumented vertebraepost-operatively. $W_{h}$ and $W_{s}$ : Weight of the head and shoulder. $M_{h}$ and $M_{s}$ : Moment caused by the weight of head and shoulder. Total bending moment $M_{\text {total }}=M_{h}+M_{s}=W_{h} \cdot L_{h} \cdot 0.8+W_{s} \cdot L_{s} \cdot 9.8$. Equivalent moment arm $L_{e}=M_{\text {total }} /\left(W_{h}+W_{s}\right)$.

Table 1: Outcomes for series of three patients undergoing surgery for kyphotic spinal deformity.

\begin{tabular}{|l|l|l|l|l|}
\hline & Case 1 & Case 2 & Case 3 & Total Avg. \\
\hline Age & 16 & 15 & 14 & $15 \pm 1$ \\
\hline Etiology & Neuromuscular & Scheuermann's & Neuromuscular & - \\
\hline Body Weight (kg) & 32.4 & 78.9 & 39.4 & $50.2 \pm 25.1$ \\
\hline Pre-op $\mathbf{M}_{\mathrm{h}}$ & 262.2 & 969.7 & 573.5 & $601.8 \pm 354.6$ \\
\hline Post-op $\mathbf{M}_{\mathrm{h}}$ & 298.4 & 513.1 & 161.9 & $324.4 \pm 177.0$ \\
\hline Final follow-up $\mathbf{M}_{\mathrm{h}}$ & 308.2 & 277.7 & 161.9 & $249.2 \pm 77.2$ \\
\hline Pre-op $\mathbf{M}_{\mathrm{s}}$ & 583.5 & 586.6 & 366.3 & $512.1 \pm 126.3$ \\
\hline Post-op $\mathbf{M}_{\mathrm{s}}$ & 572.0 & 425.9 & 189.3 & $395.7 \pm 195.1$ \\
\hline Final follow-up $\mathbf{M}_{\mathrm{s}}$ & 479.5 & 376.1 & 189.3 & $348.3 \pm 147.1$ \\
\hline Pre-op Mtota & 845.7 & 1556.4 & 939.8 & $1114.0 \pm 386.0$ \\
\hline Post-op $\mathbf{M}_{\text {total }}$ & 870.4 & 939.0 & 351.2 & $720.2 \pm 321.4$ \\
\hline Final follow-up $\mathbf{M}_{\text {total }}$ & 787.7 & 653.8 & 351.2 & $597.6 \pm 223.6$ \\
\hline Complications & PJF, loss of fixation & - & - & - \\
\hline
\end{tabular}

The total moment due to head and shoulder weight and position $M_{\text {total }}=M_{h}+M_{s}=\left(W_{h} \cdot L_{h} \cdot 9.8\right)+\left(W_{h} \cdot L_{h} \cdot 9.8\right)$ (Equation 3).

\section{Results}

Three patients underwent surgery for kyphosis (Table 1 ). Average age was $15 \pm 1$ years-old. Etiologies included one Scheuermann's kyphosis patient, and two neuromuscular kyphosis patients. Average pre-operative kyphotic deforming force atthe apex due to head and shoulder position was
$1114.0 \pm 386.0 \mathrm{~N} \cdot \mathrm{cm}$. Surgical correction decreased the deforming force at the upper instrumented vertebrae to $720.2 \pm$ $321.4 \mathrm{~N} \cdot \mathrm{cm}$ in the immediate post-operative period, and further decreased the deforming force to $597.6 \pm 223.6 \mathrm{~N} \cdot \mathrm{cm}$ at final follow-up. Only one patient (Case 1) experienced proximal junctional failure and loss of fixation. Unlike the other two cases, this patient had a persistently high kyphotic bending moment $\left(\mathrm{M}_{\text {total }}\right)$ and anterior positioning of his head and shoulders that did not improve with surgery. It is likely that 
Citation: Bonthius D, Gross R, Wu Y, et al. (2021) A Proposed Method to Quantify the Effect of Head and Shoulder Position on Kyphotic Deforming Forces before and after Spine Surgery. J Orthop Surg Tech 4(1):324-330
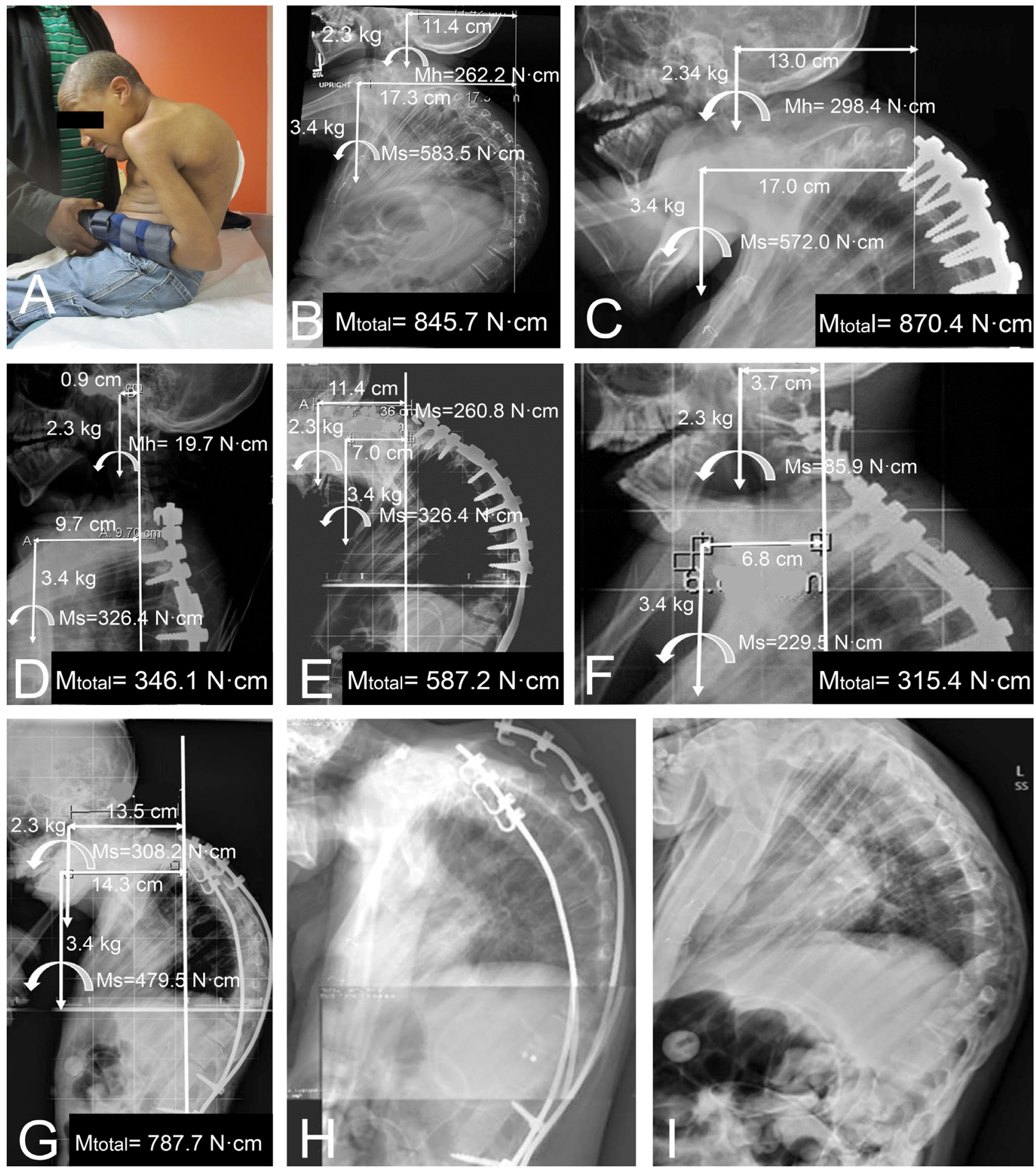

Figure 2: (A) A 16-year-old boy (Case 1) who presented with spastic quadriparesis and severe thoracic kyphosis. His shoulders were fixed in a protracted position with marked anterior positioning of his head; (B) Preoperative radiograph shows severe shoulder protraction and forward head position. There is a large kyphotic deforming force $(845.7 \mathrm{~N} \cdot \mathrm{cm})$ from the anterior head position and protracted shoulders; (C) Persistent large kyphotic deforming force $(870.4 \mathrm{~N} \cdot \mathrm{cm})$ post-operatively and immediate proximal junctional failure; (D) Immediate post-operative radiograph after extension of instrumentation to $\mathrm{C} 7$. The shoulders are still protracted; (E) Failure of revision. Increased forward position of head; (F) Failure of cervical laminar screws; (G) After hybrid rib construct inserted. Persistent anterior position of the head and protracted shoulders creating substantial kyphotic deforming force $(787.7 \mathrm{~N} \cdot \mathrm{cm}) ; \mathrm{H}$ : Failure of the instrumentation fixation on the right side; I: Instrumentation removed. Ten months later the patient's chin was resting on his chest. The cervicothoracic spine is parallel to the floor. 
Citation: Bonthius D, Gross R, Wu Y, et al. (2021) A Proposed Method to Quantify the Effect of Head and Shoulder Position on Kyphotic Deforming Forces before and after Spine Surgery. J Orthop Surg Tech 4(1):324-330

the persistently high and unimproved kyphotic bending moment in this patient contributed to his complications.

\section{Case Presentations}

\section{Case 1}

A 16-year-old boy with spastic quadriparesis presented to the orthopedic clinic with severe thoracic kyphosis. His shoulders were fixed in a protracted position with marked anterior positioning of his head (Figure 2A). Pre-operative analysis would have revealed a very large kyphotic deforming force of $845.7 \mathrm{~N} \cdot \mathrm{cm}$ (Figure 2B) and post-operative deforming force of $870.4 \mathrm{~N} \cdot \mathrm{cm}$ (Figure $2 \mathrm{C}$ ). However, his procedure was performed before the importance of head and shoulder position, as described in this paper, was appreciated.

The patient underwent corrective surgery with standard pedicle screw fixation, but had a $30^{\circ}$ proximal junctional failure when seen for follow-up two weeks later. An extension of the fusion mass to $\mathrm{C} 7$ was performed, with an initially promising result. However, the patient still had marked shoulder protraction. If kyphotic deforming forces on the upper instrumented vertebrae had been calculated at this time with the method proposed in this paper, a deforming force of 346.1
$\mathrm{N} \cdot \mathrm{cm}$ would have been appreciated, almost entirely attributable to his shoulder protraction $\left(\mathbf{M}_{\mathbf{s}}=\mathbf{3 2 6 . 4} \mathbf{N} \cdot \mathbf{c m}\right.$ ) (Figure 3D). With the marked shoulder protraction, further failure resulted (Figure 3E). Attempted salvage with cervical laminar screws failed (Figure 3F). The patient was able to hyperextend his cervical spine pre-operatively, but with his repeated posterior exposures his head was subsequently consistently forward flexed, which added to his deforming kyphotic force.

The original fixation ultimately eroded and was removed two years later. A hybrid rib construct was inserted at this time [14]. This construct used laminar hooks for proximal fixation to the ribs. Post-operative radiographs indicate a kyphotic deforming force of $787.7 \mathrm{~N} \cdot \mathrm{cm}$ (Figure 3G). Three years later, he had unilateral failure of proximal fixation (Figure 3J), and all instrumentation was removed (Figure 3I). The patient died in his sleep one year after removal of instrumentation, likely due to thoracic insufficiency.

In retrospect, this patient's pre-operative high kyphotic deforming force values could have alerted the surgeon to a high likelihood of proximal junctional failure, and pre-operative strategies to counter those forces could have been considered. For example, with his fixed shoulder protraction, a scapulopexy early in his treatment course could have reduced
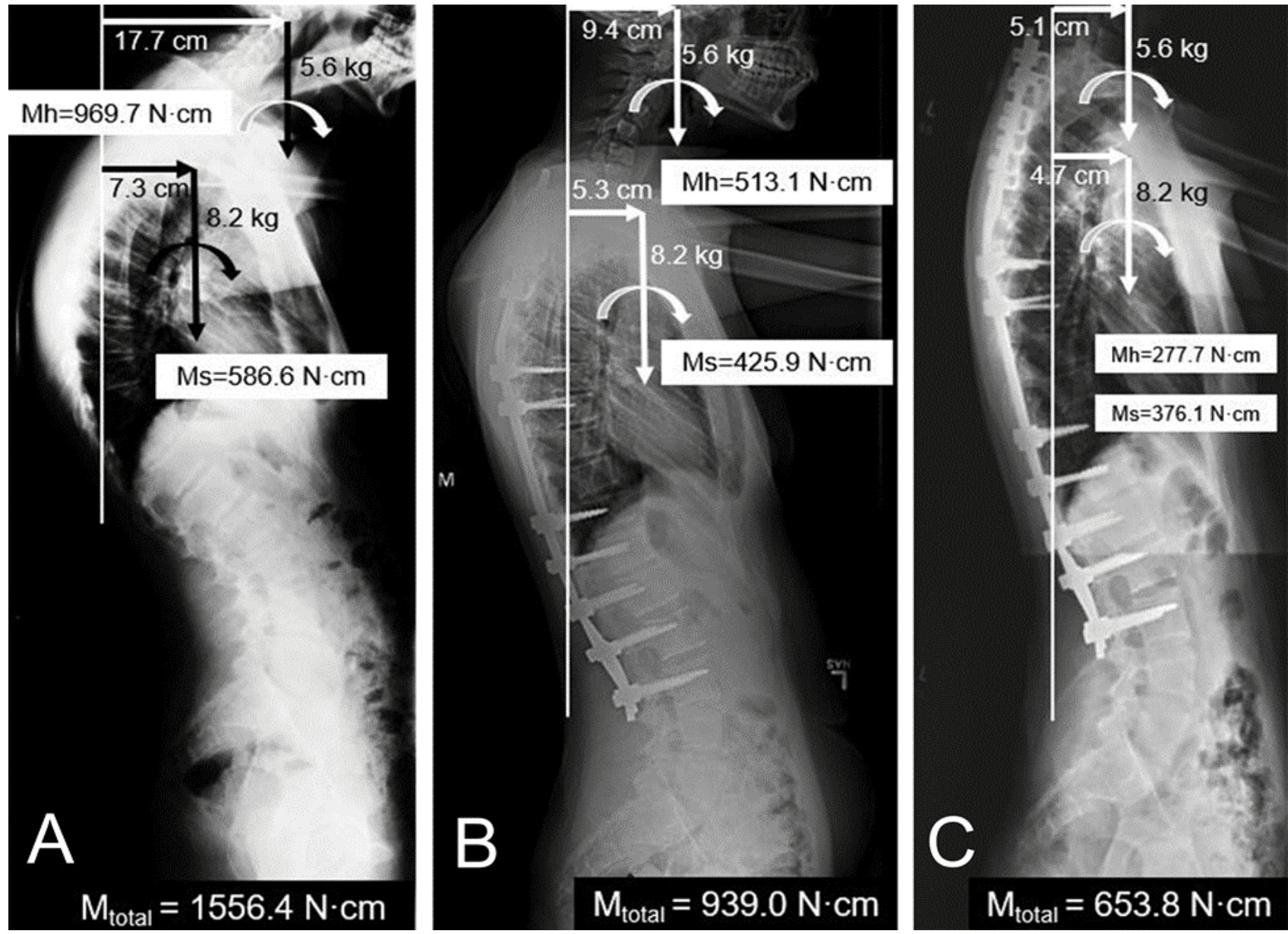

Figure 3: (A) Pre-operative kyphotic forces in a 15-year-old boy (Case 2) with Scheuermann's kyphosis and osteoporosis. Instrumentation will be subjected to considerable magnitude of kyphotic deforming forces; (B) Post-operative kyphotic forces on the upper instrumented vertebra; (C) After physical therapy, further reduction of deforming forces. 
Citation: Bonthius D, Gross R, Wu Y, et al. (2021) A Proposed Method to Quantify the Effect of Head and Shoulder Position on Kyphotic Deforming Forces before and after Spine Surgery. J Orthop Surg Tech 4(1):324-330

the persistent large moment arm of the shoulders on the fixation at the upper instrumented vertebrae.

\section{Case 2}

A 15-year-old boy presented to the orthopedic clinic with Scheuermann's kyphosis and osteoporosis (Tscore -3.4). Preoperatively, the bending moments created by his head and shoulders at the apex of his kyphotic deformity were 969.7 $\mathrm{N} \cdot \mathrm{cm}$ and $586.6 \mathrm{~N} \cdot \mathrm{cm}$, respectively, representing a substantial total kyphotic bending moment $\left(M_{\text {total }}\right)$ of $1556.4 \mathrm{~N} \cdot \mathrm{cm}$ (Figure $3 A)$. Thus, this patient was identified as being at high risk of developing proximal junctional failure or loss of proximal fixation. A hybrid rib constructwas selected for instrumentation, asanalysis in our laboratory has documented that the hybrid rib construct is superior to pedicle screw fixation in resisting kyphotic pullout forces and is especially useful in kyphotic patients with osteoporosis. Furthermore, the rib fixation avoids disruption of the posterior spinal ligaments and likely reduces the risk of proximal junctional failure. His initial postoperative radiograph revealed that his bending moment about the upper instrumented vertebrae remained high $(939.0 \mathrm{~N} \cdot \mathrm{cm})$, due to pronounced shoulder and neck protraction (Figure $3 \mathrm{~B}$ ). Fortunately, this protraction was not fixed, and it responded positively to physical therapy. Following one year of physical therapy, the total bending moment at the upper instru- mented vertebrae was reduced substantially to $653.8 \mathrm{~N} \cdot \mathrm{cm}$, a much more acceptable value (Figure $3 \mathrm{C}$ ). This boy had no post-operative complications. In particular, he did not develop proximal junctional failureor experience loss of proximal fixation over a 3-year follow-up.

\section{Case 3}

A 14-year-old girl presented with neuromuscular kyphoscoliosis secondary to spastic quadriparesis, with a similar clinical presentation to Case 1 . Her pre-operative thoracic kyphosis measured $133^{\circ}$, and her kyphotic bending moment $(939.8 \mathrm{~N} \cdot \mathrm{cm})$ was substantial (Figure 4A). She also had osteoporosis with a T score of -3.7 , which constituted a substantial risk factor for instrumentation failure. A hybrid rib construct was used to correct the patient's kyphosis, with the same rationale as in Case 2. Post-operative thoracic kyphosis measured $46^{\circ}$, and post-operative bending moment was $(351.2 \mathrm{~N} \cdot \mathrm{cm})$, which was a substantial improvement from her pre-operative value of $931.8 \mathrm{~N} \cdot \mathrm{cm}$ (Figure 4B). This patient did not experience proximal junctional failure, loss of fixation, or any other post-operative complication at three-year follow-up.

\section{Discussion}

Current assessment of sagittal balance in spinal deformity
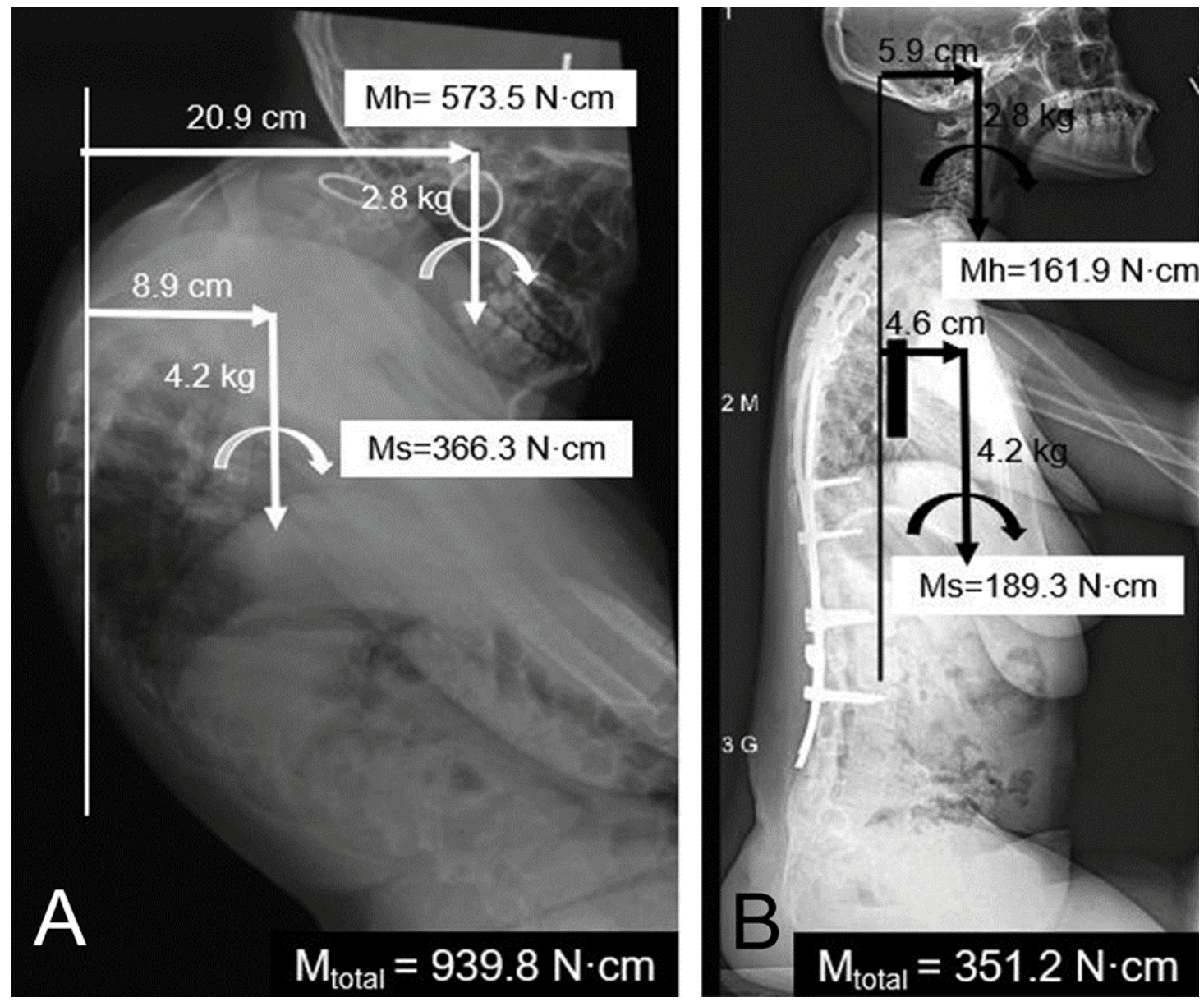

Figure 4: (A) Pre-operative and; (B) Post-operative lateral radiographs of a 14-year-old girl (Case 3) with spastic quadriparesis and secondary kyphoscoliosis. Substantial reduction in kyphotic deforming forces was achieved with surgery. 
Citation: Bonthius D, Gross R, Wu Y, et al. (2021) A Proposed Method to Quantify the Effect of Head and Shoulder Position on Kyphotic Deforming Forces before and after Spine Surgery. J Orthop Surg Tech 4(1):324-330

patients focuses primarily on the alignment of the spine and pelvis $[8,15]$. However, the kyphotic deforming forces created by head and shoulder position in some patients may be a risk factor for proximal junctional failure and loss of fixation. Here, we introduce a new radiographic measurement technique for quantifying the kyphotic deforming force of the head and shoulders on the spine.

It is likely that persistently high kyphotic deforming force values secondary to anterior head and shoulder position lead to an increased risk for proximal junctional failure and loss of fixation. This was supported by Case 1 , and clinically we have seen these outcomes in additional similar cases. For the pre-operative patient, our measurement technique could be helpful in planning surgical strategy. For the post-operative patient, the method can be used to more accurately determine the risk of complications, and the need for supplemental therapy. Consideration of the effect of head and shoulder position pre-operatively can alert the surgeon to plan a stronger construct (as was done in Cases 2 and 3), consider more proximal upper fixation, or optimize the pre-operative status with physical therapy. Post-operatively, additional head support, with a cervical collar in selected cases, and additional therapy may reduce the post-operative kyphotic deforming forces on the upper instrumented vertebrae.

The subjects in this series were all pediatric patients. However, the opportunity and value of applying this technique in adult patients with kyphosis may be even greater. For example, if an older patient has a marked forward position of the head with shoulder protraction and limited shoulder motion, then the chances for failure of fixation are substantial [16]. This is especially true if the patient has osteoporosis. Early identification of this combination of risk factors could direct the surgeon toward a pre-operative therapy program to increase back extensor strength and shoulder mobility prior to surgery. This would place less stress on the upper instrumented vertebrae, thus decreasing the likelihood of proximal junctional failure. The response of our patient in Case 2 to post-operative physical therapy illustrates its potential role in improving outcomes.

There are several limitations to the proposed measurement technique. There is a lack of normative data available to classify what normal values should be. The sample size in this study is small, like many papers describing novel radiographic techniques. The purpose of this paper is primarily to describe the technique. Future studies with a larger sample size are warranted to establish normative values and to further explore the relationship between head and shoulder position and the genesis of proximal junctional failure and loss of fixation. Also, this technique represents a simplified static biomechanical analysis of a biomechanical environment that is quite complex and dynamic. Nevertheless, this technique is a simple, fast, and informative measurement that can be performed on standard lateral radiographs. It adds tremendous value by providing a simple and useful method that will prompt the surgeon to consider head and shoulder position in kyphotic patients.

\section{Acknowledgements}

This research was supported by NIH grants F31AR076917,

\section{TL1 TR001451, and UL1 TR001450.}

\section{References}

1. Schairer WW, Carrer A, Deviren V, et al. (2013) Hospital readmission after spine fusion for adult spinal deformity. Spine 38: 1681-1689.

2. Schroerlucke SR, Akbarnia BA, Pawelek JB, et al. (2012) How does thoracic kyphosis affect patient outcomes in growing rod surgery? Spine 37: 1303-1309.

3. Reinker K, Simmons JW, Patil V, et al. (2011) Can VEPTR control progression of early-onset kyphoscoliosis? A cohort study of VEPTR $^{(\oplus)}$ patients with severe kyphoscoliosis. Clin Orthop Relat Res 469: 1342-1348.

4. Watanabe K, Uno K, Suzuki T, et al. (2013) Risk factors for complications associated with growing-rod surgery for early-onset scoliosis 38: E464-E468.

5. Lau D, Clark AJ, Scheer JK, et al. (2014) Proximal junctional kyphosis and failure after spinal deformity surgery: A systematic review of the literature as a background to classification development. Spine 39: 2093-2102.

6. Arlet V, Aebi M (2013) Junctional spinal disorders in operated adult spinal deformities: Present understanding and future perspectives. European Spine Journal 2: S276-S295.

7. Cho SK, KIm YJ, Lenke LG (2015) Proximal Junctional kyphosis following spinal deformity surgery in the pediatric patient. J AM Acad Ortho Surg 23: 408-414.

8. Lazennec JY, Brusson A, Rousseau MA (2011) Hip-spine relations and sagittal balance clinical consequences. European Spine Journal 5: 686-698.

9. Protopsaltis T, Schwab F, Bronsard N, et al. (2014) The T1 pelvic angle, a novel radiographic measure of global sagittal deformity, accounts for both spinal inclination and pelvic tilt and correlates with health-related quality of life. The Journal of Bone and Joint Surgery American volume 96: 1631-1640.

10. Imagama S, Hasegawa Y, Wakao N, et al. (2014) Impact of spinal alignment and back muscle strength on shoulder range of motion in middle-aged and elderly people in a prospective cohort study. European Spine Journal 23: 1414-1419.

11. Kebaetse M, McClure P, Pratt NA (1999) Thoracic position effect on shoulder range of motion, strength, and three-dimensional scapular kinematics. Arch Phys Med Rehabil 80: 945-950.

12. Smith J, Dietrich CT, Kotajarvi BR, et al. (2006) The effect of scapular protraction on isometric shoulder rotation strength in normal subjects. Journal of Shoulder and Elbow Surgery 15: 339343.

13. Clauser CE, McConville JT, Young JW (1969) Weight, volume, and center of mass of segments of the human body. Wright-Patterson Air Force Base: Aerospace medical research laboratory.

14. Gross RH (2012) An alternate method of fixation for management of early-onset deformity with thoracic kyphosis. J Pediatr Orthop 32: e30-e34.

15. Schwab F, Lafage V, Boyce R, et al. (2006) Gravity line analysis in adult volunteers: Age-related correlation with spinal parameters, pelvic parameters, and foot position. Spine 31: e959-e967.

16. Mika A, Unnithan VB, Mika P (2005) Differences in thoracic kyphosis and back muscle strength in women with bone loss due to osteoporosis. Spine 30: 241-246. 